\title{
Fatores associados ao contato pele a pele imediato em uma maternidade
}

\author{
Factors associated with early skin-to-skin contact in a maternity hospital \\ Factores asociados con el contacto inmediato piel a piel en una maternidad
}

\begin{abstract}
Lilian Fernandes Arial Ayres ${ }^{1}$ (1) Raquel Elisabeth Cnossen ${ }^{2}$ (a) Camila Mendes dos Passos ${ }^{1}$ (C) Vanessa Doriguêtto Lima $^{3}$ (C) Mara Rúbia Maciel Cardoso do Prado ${ }^{1}$ (1) Brenda Alves Beirigo 4 [D
\end{abstract}

1. Universidade Federal de Viçosa. Viçosa, MG, Brasil.

2. Universidade de Brasília. Brasília, DF, Brasil. 3. Secretaria Municipal de Saúde. Viçosa MG, Brasil.

4. Hospital Sofia Feldman. Belo Horizonte, $M G$, Brasil.
Autor correspondente Lilian Fernandes Arial Ayres E-mail: lilian.ayres@ufv.br

Recebido em 20/04/2020.

Aprovado em 30/09/2020.

DOl:https://doi.org/10.1590/2177-9465-EAN-2020-0116

\section{RESUMO}

Objetivo: Estimar a ocorrência do contato pele a pele imediato e sua associação aos fatores sociodemográficos, obstétricos, assistenciais e de nascimento em uma maternidade da Zona da Mata Mineira. Método: Estudo transversal realizado com 222 primíparas por meio de entrevista e dados do prontuário. Os dados foram codificados, categorizados, digitados e analisados pelo programa Epi info 7.0. Utilizou-se a regressão logística múltipla. Resultados: A ocorrência do contato pele a pele imediato foi de $30 \%$ e foi associado ao: profissional do parto não ser o mesmo do pré-natal (OR 3,17; IC 95\% 1,52 -6,62), presença de acompanhante (OR 3,35; IC 95\% 1,67-6,73) e realização de parto normal (OR 15,59; IC 95\% 7,50-32,41). Conclusão e implicações para a prática: É primordial incentivar o parto normal, sensibilizar profissionais e empoderar as mulheres sobre o direito do acompanhante e contato pele a pele, pois este minimiza as intervenções na primeira hora, estimula o vínculo e promove a amamentação.

Palavras-chaves: Aleitamento Materno; Período Pós-Parto; Recém-Nascido.

\begin{abstract}
Objective: To estimate the prevalence of early skin-to-skin contact and its association with sociodemographic, obstetric, assistance and birth factors in a maternity located in the Forest Zone of Minas Gerais (southeast Brazil). Method: A cross-sectional study was carried out with 222 primiparous women, by means of interview and data from the medical records. The data were coded categorized, typed and analyzed using the Epi info 7.0 software. Multiple logistic regression was used. Results: The occurrence of skin-to-skin contact was $30 \%$ and was associated with: professional delivery not being the same as prenatal care (OR 3.17 95\% Cl 1.52 -6.62), presence of companion (OR 3.35; 95\% Cl 1.67-6.73) and normal delivery (OR 15.59; 95\% Cl 7.50-32.41). Conclusion and implications for practice: It is essential to encourage normal childbirth, sensitize professionals and empower women about the right of the companion and skin-to-skin contact, as this minimizes interventions in the first hour, stimulates mother-baby bond and promotes breastfeeding
\end{abstract}

Keywords: Breast Feeding. Postpartum Period. Infant, Newborn.

\section{RESUMEN}

Objetivo: Estimar la prevalencia del contacto inmediato piel a piel y su asociación con factores sociodemográficos, obstétricos asistenciales y de nacimiento en una sala de maternidad en la Zona de la Mata Minera (Brasil). Método: Estudio transversal realizado con 222 mujeres primíparas, a través de entrevistas y datos de registros médicos. Los datos fueron codificados, categorizados, tipificados y analizados por el programa Epi info 7.0. Se utilizó la regresión logística múltiple. Resultados: La incidencia del contacto inmediato piel a piel fue del $30 \%$ y se asoció con: profesional del parto que no es lo mismo que de la asistencia prenatal (OR 3.17; IC del 95\% 1.52 -6.62), presencia de acompañante (OR 3.35; IC 95\% 1.67-6.73) y parto normal (OR 15.59; IC 95\% 7.50-32.41). Conclusión e implicaciones para la práctica: Es esencial fomentar el parto normal, sensibilizar a los profesionales y empoderar a las mujeres sobre el derecho del acompañante y el contacto piel a piel, ya que esto minimiza las intervenciones en la primera hora, estimula el vínculo y promueve la lactancia materna.

Palabras clave: Lactancia Materna; Periodo Posparto; Recién Nacido. 


\section{INTRODUÇÃO}

A assistência imediata ao recém-nascido $(R N)$ a termo na sala de parto sofreu um processo de transformação nos últimos anos. ${ }^{1} \mathrm{~A}$ qualidade dessa assistência vem sendo um dos principais meios para diminuir a taxa de mortalidade neonatal, que atualmente é responsável por quase $70 \%$ dos casos de óbito no primeiro ano de vida. Em 2011, o Ministério da Saúde criou a Rede Cegonha, visando qualificar a assistência materno-infantil, e um dos objetivos foi assegurar a incorporação de boas práticas ao RN. ${ }^{2}$

As boas práticas de cuidado com o $\mathrm{RN}$ também visam à redução do número de procedimentos dirigidos a ele, a fim de proporcionar maior interação entre mãe e bebê sobretudo na primeira hora de vida. ${ }^{1}$ Entre os cuidados imediatos recomendados na primeira hora de vida estão a presença do acompanhante, ambiência, sonoridade, luminosidade, temperatura do ambiente, clampeamento do cordão umbilical tardio, prevenção da perda do calor do recém-nascido (secado e aquecido no ventre materno), contato pele a pele precoce e amamentação durante a primeira hora de vida. Estes cuidados sofrem variações nos serviços obstétricos brasileiros, sendo mais prevalente em Centro de Parto Normal ou Casa de Parto que se apoiam nos princípios da integralidade e da humanização do parto e contam com a presença da enfermagem obstétrica na atenção ao parto e nascimento com valorização do processo fisiológico da parturição e nascimento. ${ }^{3} \mathrm{O}$ contato pele a pele precoce e contínuo deve ser realizado sem roupa, em decúbito ventral sobre o tórax da mãe, entre os seios, também despidos, logo após o nascimento ${ }^{4} \mathrm{e}$ por 1 hora. ${ }^{5}$ Esta prática compõe o quarto passo dentre os dez instituídos na política de incentivo à amamentação (Iniciativa Hospital Amigo da Criança - IHAC). ${ }^{5}$

Fatores hormonais, sensoriais, fisiológicos, imunológicos e comportamentais são estimulados através do contato pele a pele. Essa boa prática produz benefícios, como: fortalece o vínculo, incentiva o aleitamento materno, ${ }^{6}$ estimula o cuidar materno, proporciona alívio e tranquilidade para mãe e bebê, promove e regula a temperatura do bebê ${ }^{6}$, preserva o equilíbrio acidobásico, estabiliza a respiração, reduz o choro, ajuda a colonização bacteriana pela microbiota cutânea da mãe, previne a hipoglicemia neonatal, melhora os parâmetros neurocomportamentais na infância e facilita o relacionamento, ${ }^{7,8}$ auxilia a expulsão da placenta e reduz as chances de intercorrências no pós-parto como a hemorragia após o parto. ${ }^{9}$

Apesar desses benefícios e das atuais políticas de saúde direcionadas ao RN, segundo a pesquisa Nascer no Brasil, âmbito nacional, em 2011 e 2012, apenas $28,2 \%$ dos bebês tiveram contato pele a pele com a mãe após o nascimento. ${ }^{1}$ Compreende-se que a prioridade continua sendo os cuidados ou as intervenções imediatas na maioria das vezes desnecessárias ao $\mathrm{RN}$, como aspiração de vias aéreas, secção do cordão umbilical, profilaxia ocular, dentre outras, e não a assistência humanizada. Pressupõe-se que esses procedimentos ocorram devido à rotina das maternidades, à agilidade na assistência e à necessidade de desocupar os leitos obstétricos. ${ }^{10}$ Neste contexto, este estudo objetiva estimar a ocorrência do contato pele a pele imediato e sua associação com fatores sociodemográficos, obstétricos, assistenciais e de nascimento em primíparas e seus bebês em uma maternidade de um município da Zona da Mata de Minas Gerais.

\section{MÉTODO}

O presente estudo foi aprovado pelo Comitê de Ética e Pesquisa com seres humanos da Universidade Federal, estando de acordo com a resolução no 466/12 do Conselho Nacional de Saúde. As entrevistas foram realizadas por uma equipe treinada com todas as primíparas que aceitaram participar do estudo, após apresentação do Termo de Consentimento Livre e Esclarecido (TCLE).

Trata-se de estudo transversal, que faz parte de uma pesquisa maior intitulada "Fatores determinantes para a decisão da via de parto em primíparas em uma maternidade do município da Zona da Mata Mineira". O cenário do estudo foi a única maternidade de um município que possui uma população em torno de 80 mil habitantes. Esta instituição é credenciada como Hospital de Ensino de uma Universidade Federal e oferece atendimento para o Sistema Único de Saúde (SUS), particular e convênios. A coleta de dados ocorreu de novembro de 2015 a outubro de 2016, no período diurno, durante os setes dias da semana.

A população do estudo foi composta de puérperas primíparas internadas na maternidade por ocasião do parto. Os critérios de inclusão foram primíparas de parto vaginal ou cesárea, com feto vivo ao início do trabalho de parto. O critério de exclusão foi a impossibilidade da realização do procedimento em virtude de uma infecção contagiosa. Para o cálculo amostral, considerouse uma população de 739 primíparas no ano de 2014, sendo a frequência de cesárea $71 \%$ e de partos normais 29\% (dados obtidos no livro de parto da instituição). O nível de significância estabelecido foi de $95 \%$ e o erro tolerável de $5 \%$. A amostra foi composta por 222 puérperas primíparas.

Os dados primários foram obtidos através da aplicação de um questionário estruturado às puérperas durante as primeiras 24 horas após o parto, e os dados secundários foram retirados do prontuário por meio de um questionário semelhante ao aplicado à puérpera. Esses instrumentos contêm diversas informações relacionadas ao perfil sociodemográfico e obstétrico, à assistência obstétrica, às condições de nascimento e às características RN.

A variável dependente (contato pele a pele) foi verificada a partir da pergunta: "O RN foi colocado em contato pele a pele com você na sala de parto?”. Após a pergunta, as entrevistadoras explicaram como deve ser esse procedimento. Os fatores investigados (variáveis independentes) como potencialmente associados à realização do contato pele a pele foram: idade materna em anos, município da maternidade e região, cor da pele autorreferida (branca, negra, parda), escolaridade média em anos (desvio-padrão), situação conjugal (solteira, casada, união consensual), trabalho remunerado (sim, não), renda em salário mínimo, renda familiar em salário mínimo, gestação planejada (sim, não), financiamento pré-natal (SUS, particular), número de consultas do pré-natal, problema de saúde prévio (sim, não), problema de saúde desenvolvido na gestação (sim, não), intercorrências na gestação (sim, não), via de parto (normal, cesárea), mesmo profissional no pré-natal e no parto (sim, não, não soube informar), acompanhante (sim, não, não soube 
informar), problemas no parto (sim, não, não soube informar), parto traumático para o RN (sim, não, sem informação), valor do Apgar no primeiro minuto, valor do Apgar no quinto minuto, peso ao nascer em gramas, malformação fetal (sim, não, sem informação) e RN encaminhado para (berçário, UTI, alojamento conjunto, não soube informar).

$\mathrm{Na}$ análise descritiva das variáveis foram estimados as frequências absolutas e relativas, as médias, os desvios-padrão e os intervalos interquartílicos, com o intuito de caracterizar a população estudada quanto às variáveis do estudo. Em seguida, foi conduzida a análise inferencial (analítica) para identificar os fatores associados ao desfecho estudado (realização do contato pele a pele). Para verificar as associações entre as variáveis categóricas, utilizou-se o teste qui-quadrado de Pearson. Nas variáveis contínuas foi testada a normalidade da distribuição, utilizando-se o teste Kolmogorov Smirnov. Utilizou-se o teste paramétrico ( $t$ de Student) ou não paramétrico (Mann-Whitney), de acordo com o resultado do teste de normalidade. Para todos os testes foi fixado o nível de confiança de $95 \%$.

A associação entre o contato pele a pele e as variáveis explicativas foi realizada a partir do modelo de regressão logística binária e múltipla. Para a seleção do modelo final da regressão logística, empregou-se o método de eliminação backward por razão de verossimilhança (LR). Ele começa com a inclusão de todas as variáveis explicativas significativas no modelo $(p<0,20)$ na análise bivariável. As variáveis são, então, retiradas uma de cada vez, começando com a que reduz a LR pela mínima quantidade. A equação é avaliada em cada etapa e o procedimento é repetido até que cada variável que permanece no modelo explique uma porção significativa da variação observada na resposta. ${ }^{11}$ No modelo multivariável foram consideradas significativas as variáveis que apresentaram $p<0,05$. Para avaliar a magnitude das associações, foram usados o odds ratio e seus respectivos intervalos de confiança de $95 \%$. Todas as informações foram compiladas e integradas em um único banco de dados. Os dados coletados foram codificados, categorizados, digitados e analisados em um banco do programa Epi info 7.0.

\section{RESULTADOS}

Apenas 30\% dos bebês foram colocados em contato pele a pele imediato e, mesmo contando com a mediana do Apgar do primeiro e do quinto minuto em torno de $9 \mathrm{e} 10$, respectivamente; a média do peso ao nascer foi $3,2 \mathrm{~kg}$. Além disso, a maioria dos RNs não sofreu parto traumático e foi encaminhado ao alojamento conjunto (Tabela1).

$\mathrm{Na}$ análise bivariada, a frequência do contato pele a pele imediato foi maior entre as primíparas que não foram assistidas pelo mesmo profissional do pré-natal, com acompanhante na sala de parto e entre as que realizaram o parto normal. Devese destacar que as mulheres submetidas ao parto normal apresentaram maior diferença de frequência (Tabela 2).

Na análise bruta, as variáveis: profissional que fez o parto foi o mesmo do pré-natal; acompanhante na sala de parto e via de parto

Tabela 1. Características do nascimento de mulheres primíparas entrevistadas na maternidade do município da Zona da Mata, Minas Gerais, Brasil, 2015-2016.

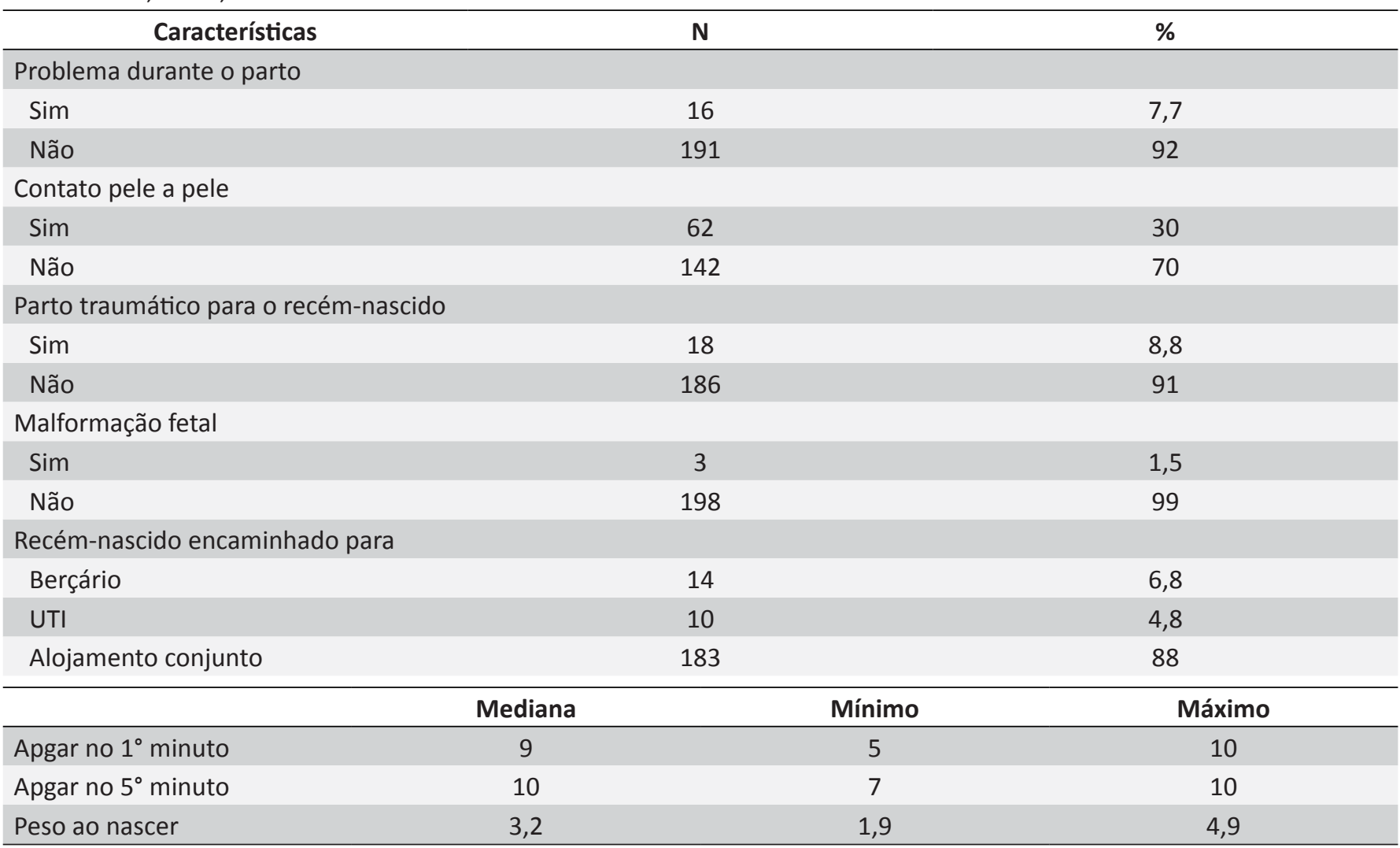


Tabela 2. Características sociodemográficas, obstétricas, assistenciais e de nascimento segundo a realização do contato pele a pele em primíparas entrevistadas na maternidade do município de Zona da Mata, Minas Gerais, Brasil, 2015-2016.

\begin{tabular}{|c|c|c|c|c|c|}
\hline \multirow{3}{*}{ Características maternas } & \multicolumn{4}{|c|}{ Contato pele a pele } & \multirow{3}{*}{ Valor de $\mathrm{p}^{*}$} \\
\hline & \multicolumn{2}{|c|}{ Sim } & \multicolumn{2}{|c|}{ Não } & \\
\hline & $\mathbf{N}$ & $\%$ & $\mathbf{N}$ & $\%$ & \\
\hline \multicolumn{6}{|l|}{ Município } \\
\hline Município da Maternidade & 35 & 30,4 & 80 & 69,6 & $0,988^{b}$ \\
\hline Outros & 27 & 30,3 & 62 & 69,7 & \\
\hline \multicolumn{6}{|l|}{ Cor da pele } \\
\hline Branca & 15 & 24,6 & 46 & 75,4 & \\
\hline Negra & 14 & 32,6 & 29 & 67,4 & $0,533^{b}$ \\
\hline Parda & 31 & 32,3 & 65 & 67,7 & \\
\hline \multicolumn{6}{|l|}{ Situação conjugal } \\
\hline Solteira & 19 & 32,2 & 40 & 67,8 & \\
\hline Casada & 32 & 27,3 & 85 & 72,7 & $0,373^{b}$ \\
\hline União consensual & 11 & 39,3 & 17 & 60,7 & \\
\hline \multicolumn{6}{|l|}{ Trabalho renumerado } \\
\hline Sim & 29 & 26,1 & 82 & 73,9 & $0,148^{b}$ \\
\hline Não & 33 & 35,5 & 60 & 64,5 & \\
\hline \multicolumn{6}{|l|}{ Gestação planejada } \\
\hline Sim & 38 & 29,2 & 92 & 70,8 & $0,632^{\mathrm{b}}$ \\
\hline Não & 24 & 32,4 & 50 & 67,6 & \\
\hline \multicolumn{6}{|l|}{ Financiamento do pré-natal } \\
\hline SUS & 40 & 32,3 & 84 & 67,7 & $0,470^{b}$ \\
\hline Particular & 22 & 27,5 & 58 & 72,5 & \\
\hline \multicolumn{6}{|l|}{ Problema de saúde prévio } \\
\hline Sim & 13 & 40,6 & 19 & 59,4 & $0,170^{\mathrm{b}}$ \\
\hline Não & 49 & 28,5 & 123 & 71,5 & \\
\hline \multicolumn{6}{|l|}{ Problema de saúde desenvolvido na gestação } \\
\hline Sim & 27 & 27 & 73 & 73 & $0,301^{b}$ \\
\hline Não & 35 & 33,7 & 69 & 66,3 & \\
\hline \multicolumn{6}{|l|}{ Intercorrência na gestação } \\
\hline Sim & 4 & 23,5 & 13 & 76,5 & $0,520^{\mathrm{b}}$ \\
\hline Não & 58 & 31 & 129 & 69 & \\
\hline \multicolumn{6}{|l|}{ Via de parto } \\
\hline Normal & 43 & 70,5 & 18 & 29,5 & $<0,01^{b *}$ \\
\hline Cesárea & 19 & 13,3 & 124 & 86,7 & \\
\hline \multicolumn{6}{|c|}{ Profissional que fez o parto foi o mesmo do pré-natal } \\
\hline Sim & 14 & 20 & 56 & 80 & $0,018^{b}$ \\
\hline Não & 48 & 36,1 & 85 & 63,9 & \\
\hline \multicolumn{6}{|l|}{ Acompanhante na sala de parto } \\
\hline Sim & 45 & 37,5 & 75 & 62,5 & $0,009^{b}$ \\
\hline Não & 17 & 20,5 & 66 & 79,5 & \\
\hline \multicolumn{6}{|l|}{ Problemas durante o parto } \\
\hline Sim & 2 & 12,5 & 14 & 87,5 & $0,105^{b}$ \\
\hline Não & 60 & 31,9 & 128 & 68,1 & \\
\hline \multicolumn{6}{|l|}{ Parto traumático para o recém-nascido } \\
\hline Sim & 3 & 21,4 & 11 & 78,6 & $0,469^{b}$ \\
\hline Não & 53 & 30,6 & 120 & 69,4 & \\
\hline Malformação fetal & & & & & \\
\hline Sim & 2 & 40 & 3 & 60 & $0,658^{b}$ \\
\hline Não & 55 & 30,7 & 124 & 69,3 & \\
\hline Recém-nascido encaminhado para & & & & & \\
\hline Berçário & 4 & 28,6 & 10 & 71,4 & \\
\hline UTIN & 3 & 30 & 7 & 70 & $0,984^{b}$ \\
\hline Alojamento conjunto & 55 & 30,6 & 125 & 69,4 & \\
\hline
\end{tabular}

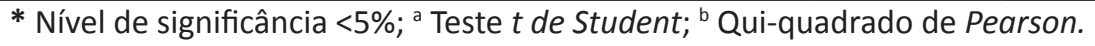


Tabela 2. Continuação...

\begin{tabular}{|c|c|c|c|c|c|}
\hline \multirow{2}{*}{ Características maternas } & \multicolumn{2}{|c|}{ Sim } & \multicolumn{2}{|c|}{ Não } & \multirow{2}{*}{ Valor de $\mathrm{p}^{*}$} \\
\hline & Média & DP & Média & DP & \\
\hline Idade materna (em anos) & 25,76 & 6,1 & 25,8 & 5,9 & $0,960^{\mathrm{a}}$ \\
\hline Escolaridade (em anos) & 11,48 & 3,5 & 11,38 & 3,4 & $0,841^{a}$ \\
\hline Renda média em salário mínimo (DP) & 0,71 & 1,1 & 0,82 & 1,2 & $0,520^{\mathrm{a}}$ \\
\hline Renda familiar média em salário mínimo (DP) & 2,14 & 2,2 & 2,53 & 3,6 & $0,436^{a}$ \\
\hline Número de consultas pré-natal (DP) & 8,38 & 2,9 & 8,71 & 2,7 & $0,458^{a}$ \\
\hline Apgar no $1^{\circ}$ minuto (DP) & 7,73 & 2,8 & 7,32 & 2,9 & $0,361^{\text {a }}$ \\
\hline Apgar no $5^{\circ}$ minuto (DP) & 8,97 & 2,5 & 8,49 & 3 & $0,270^{\mathrm{a}}$ \\
\hline Peso ao nascer (DP) & 3,18 & 0,4 & 3,23 & 0,4 & $0,460^{\mathrm{a}}$ \\
\hline
\end{tabular}

* Nível de significância $<5 \%$; ${ }^{\text {T Teste }} t$ de Student; ${ }^{\text {b }}$ Qui-quadrado de Pearson.

Tabela 3. Valores de odds ratio brutos e ajustados da associação entre contato pele a pele e características sociodemográficas, obstétricas, assistenciais e de nascimento de primíparas entrevistadas na maternidade do município da Zona da Mata, Minas Gerais, Brasil, 2015-2016

\begin{tabular}{|c|c|c|c|c|}
\hline \multirow{2}{*}{ Características maternas } & \multicolumn{2}{|c|}{ Análise bruta } & \multicolumn{2}{|c|}{ Análise ajustada } \\
\hline & OR & IC95\% & OR & IC95\% \\
\hline \multicolumn{5}{|l|}{ Trabalho renumerado } \\
\hline Sim & 1 & - & - & - \\
\hline Não & 1,56 & $0,85-2,86$ & - & - \\
\hline \multicolumn{5}{|l|}{ Problema de saúde prévio } \\
\hline Sim & 1,71 & $0,78-3,74$ & - & - \\
\hline Não & 1 & - & - & - \\
\hline \multicolumn{5}{|l|}{ Via de parto } \\
\hline Normal & 15,59 & $7,50-32,41$ & 15,59 & $7,50-32,41$ \\
\hline Cesárea & 1 & - & 1 & - \\
\hline \multicolumn{5}{|c|}{ Profissional que fez o parto foi o mesmo do pré-natal } \\
\hline Sim & 1 & - & - & - \\
\hline Não & 2,27 & $1,14-4,55$ & - & - \\
\hline \multicolumn{5}{|l|}{ Acompanhante na sala de parto } \\
\hline $\operatorname{Sim}$ & 2,33 & $1,22-4,46$ & - & - \\
\hline Não & 1 & - & - & - \\
\hline \multicolumn{5}{|l|}{ Problema durante o parto } \\
\hline Sim & 1 & - & - & - \\
\hline Não & 3,34 & $0,72-14,29$ & - & - \\
\hline
\end{tabular}

permaneceram significativas quanto à realização do contato pele a pele precoce. As primíparas que não realizaram o parto com o mesmo profissional do pré-natal e possuíam acompanhante tiveram aproximadamente duas vezes mais chance, respectivamente, de realizar o contato pele a pele imediato. As mulheres que realizaram parto normal tiveram cerca de 15 vezes mais chance de vivenciar essa boa prática, quando comparadas com as que passaram por cesárea. Após a análise ajustada, a única variável que permaneceu foi a via de parto (Tabela 3).

Destaca-se que, após a regressão logística, a variável - via de parto apresenta-se como possível fator confundidor. Por isso, optou-se por sua exclusão e por uma nova regressão logística. Desse modo, permaneceram as variáveis: não realizaram o parto com o mesmo profissional do pré-natal e acompanhante, que tiveram em torno de três vezes mais chance de realizar o contato pele a pele imediato após a análise ajustada (Tabela 4).

\section{DISCUSSÃO}

A ocorrência do contato pele a pele imediato nesta amostra de primíparas encontra-se aquém do que é preconizado pela OMS para um Hospital Amigo da Criança (HAC), pois ele deve ser realizado em pelo menos $80 \%$ das mães de parto vaginal ou cesárea sem anestesia geral. ${ }^{12}$ Embora a maternidade deste estudo ainda não seja um HAC, ela está em processo de tornar-se, portanto, a execução do quarto passo da IHAC é uma iniciativa essencial para a consolidação desse projeto. 
Tabela 4. Valores de odds ratio brutos e ajustados da associação entre contato pele a pele e trabalho renumerado, problema de saúde prévio, mesmo profissional, acompanhante e problema durante o parto de entrevistadas na maternidade do município da Zona da Mata, Minas Gerais, Brasil, 2015-2016.

\begin{tabular}{|c|c|c|c|c|}
\hline \multirow{2}{*}{ Características maternas } & \multicolumn{2}{|c|}{ Análise bruta } & \multicolumn{2}{|c|}{ Análise ajustada } \\
\hline & OR & IC95\% & OR & IC95\% \\
\hline \multicolumn{5}{|l|}{ Trabalho renumerado } \\
\hline Sim & 1 & - & - & - \\
\hline Não & 1,56 & $0,85-2,86$ & - & - \\
\hline \multicolumn{5}{|l|}{ Problema de saúde prévio } \\
\hline Sim & 1,71 & $0,78-3,74$ & - & - \\
\hline Não & 1 & - & - & - \\
\hline \multicolumn{5}{|c|}{ Profissional que fez o parto foi o mesmo do pré-natal } \\
\hline Sim & 1 & - & 1 & - \\
\hline Não & 2,27 & $1,14-4,55$ & 3,17 & $1,52-6,62$ \\
\hline \multicolumn{5}{|l|}{ Acompanhante na sala de parto } \\
\hline Sim & 2,33 & $1,22-4,46$ & 3,35 & $1,67-6,73$ \\
\hline Não & 1 & - & 1 & - \\
\hline \multicolumn{5}{|l|}{ Problema durante o parto } \\
\hline Sim & 1 & - & 1 & - \\
\hline Não & 3,34 & $0,72-14,29$ & 3,84 & $0,81-18,30$ \\
\hline
\end{tabular}

A ocorrência do contato pele a pele nessa maternidade foi similar à do resultado encontrado na pesquisa Nascer no Brasil ${ }^{1} \mathrm{e}$ foi bem menor que no estudo realizado no Nordeste Brasileiro, onde foi de $41,4 \%{ }^{13}$ e $93,2 \%$ dos bebês foram colocados em contato pele a pele imediato com as mães. Neste último estudo, os partos foram assistidos por enfermeira (o) obstetra. ${ }^{14} \mathrm{~A}$ menor ocorrência do contato pele a pele pode estar relacionada ao fato de esta pesquisa avaliar apenas as primíparas, visto que em um estudo ${ }^{1}$ encontrou-se maior número de intervenções realizadas na primeira hora de vida em RNs de primíparas, do qual pressupõe-se que essas mulheres sejam mais inseguras, sendo mais propensas a aceitar as recomendações do profissional de saúde devido à ausência de informação e pela falta da experiência de parir.

Os fatores sociodemográficos não foram uma associação significativa com a realização do contato pele a pele. Outro estudo também não relacionou significativamente fatores individuais, como idade, raça, escolaridade, estado civil e situação socioeconômica com a realização dessa prática..$^{15}$ Destaca-se que o financiamento da maternidade e o tipo de parto a que são submetidas podem influenciar a execução do contato pele a pele, uma vez que há maior investimento da implantação da HAC em maternidades públicas, ${ }^{16} \mathrm{e}$ mulheres com menor poder aquisitivo são mais propensas a realizar parto normal. ${ }^{17} \mathrm{Em}$ contrapartida, um estudo mostrou maior dificuldade de adesão no Nordeste do País, mesmo com a IHAC consolidada no local. ${ }^{15} \mathrm{Em}$ outro estudo constatou-se que mulheres provenientes do interior, com baixa escolaridade e financiamento pelo SUS possuíam menor chance de contato pele a pele. ${ }^{1}$

Geralmente, as mulheres depositam maior confiança no profissional de saúde que as acompanha no pré-natal e no parto, ${ }^{18}$ por causa da maior vinculação entre eles, ${ }^{19}$ portanto, cabe a eles oferecer orientações conforme as evidências científicas. ${ }^{18,19}$ Sendo assim, espera-se que a realização do contato pele a pele após o parto ocorra com maior frequência nesse grupo de mulheres, resultado contrário ao encontrado neste estudo. E ainda, destaca-se que o financiamento destas mulheres é por convênio ou particular.

A associação da presença do acompanhante à realização do contato pele a pele foi observada neste estudo. Certifica-se que o acompanhante favorece a humanização da assistência obstétrica e neonatal, diminui as intervenções desnecessárias e contribui para a realização das boas práticas. Ele estimula a interação precoce do binômio, aumenta o vínculo e permite que as mulheres fiquem mais tranquilas e seguras em ter o $\mathrm{RN}$ em seu colo na primeira hora de vida. ${ }^{1,8}$

Sabe-se que todo RN ativo e de baixo risco deve ser colocado em contato pele a pele com a mãe logo após o parto, permanecendo ali na sua primeira hora de vida. Exames e procedimentos de rotina só devem ser realizados após o estabelecimento desse contato, exceto em caso de indicação médica. ${ }^{3}$ Entretanto, neste estudo, apesar de $92,3 \%$ dos partos não terem apresentado problemas 
e a maioria dos bebês apresentar índice de Apgar em torno de 9 e 10 no primeiro e quinto minuto de vida, respectivamente, houve baixa ocorrência de contato pele a pele.

Os resultados permitiram associar uma variável bastante significativa à realização do contato pele a pele, a via de parto. Outros estudos ${ }^{1,8,15,19,20}$ confirmam essa associação, evidenciando que há maior dificuldade na efetuação dessa prática quando o nascimento se dá por cesárea. Nesta amostra, a mãe que fez parto normal teve uma chance, aproximadamente, 15 vezes maior de realizar o contato pele a pele do que àquela que teve parto cesárea. Elencam-se como obstáculos o retardo na interação após o nascimento, a diminuição do estado alerta do bebê, o fato de as mães estarem mais sonolentas devido à analgesia, o número reduzido de profissionais, a maca estreita, ${ }^{20}$ a recusa dos anestesistas e obstetras ${ }^{8,20}$ e o fato de frequentemente não se permitir o direito da presença do acompanhante durante a cesárea.

Nota-se que o baixo cumprimento do quarto passo da IHAC está diretamente relacionado com o modelo obstétrico vigente no Brasil que é marcado por intervenções desnecessárias e medicalizado. ${ }^{15}$ Adiciona-se que as altas taxas de cesárea dificultam a realização dessa boa prática. ${ }^{15}$ Embora o parto cesárea seja uma situação dificultadora para a realização do contato, compreende-se que as boas práticas devem ser realizados em ambos os tipos de partos. ${ }^{6,11}$

Acredita-se que a transformação do cenário da pesquisa envolve a mudança de atitude de todos os profissionais de saúde e o empoderamento das mulheres, iniciando no atendimento do pré-natal com ações educativas e preventivas até os serviços oferecidos pela maternidade. A inclusão de uma (o) enfermeira (o) obstétrica na assistência ao ciclo gravídico puerperal sobretudo na maternidade pode auxiliar essa modificação, pois esse profissional compreende o parto como um evento fisiológico, contribui para a humanização do parto e nascimento, rompe com o parto medicalizado e reduz as chances de intervenções desnecessárias. ${ }^{14}$

Apesar da existência de políticas públicas de saúde como Rede Cegonha e IHAC que incentivam o contato pele a pele imediato, observa-se a baixa ocorrência desta boa prática no país, através da pesquisa - Nascer no Brasil - e no município de estudo. Considerando os diversos benefícios advindos desta prática, este trabalho contribui para reforçar a importância do estabelecimento de estratégias que visem a implementação destas políticas, a fim de melhorar e qualificar a assistência ofertada ao binômio mãe-bebê.

\section{CONCLUSÕES}

Identificaram-se os fatores limitantes relacionados com a não realização do contato pele a pele imediato entre mãe e bebê: o profissional que fez o pré-natal ser o mesmo que realizou o parto, mulheres sem a presença de acompanhante durante o parto e mulheres que foram submetidas ao parto cesárea. Desta forma, deve-se promover o incentivo ao parto normal, a fim de reduzir as altas taxas de cesárea, sensibilizar os profissionais médicos e outros profissionais de saúde envolvidos na assistência ao pré-natal, parto e pós-parto e estimular as boas práticas em todos os cenários obstétricos, inclusive nos centros cirúrgicos.

Os profissionais de saúde que estão envolvidos na assistência obstétrica precisam apoiar de maneira concreta a prática do contato pele a pele, em virtude de suas inúmeras vantagens e para beneficiar um maior número de mães e bebês. Propõese que por meio da educação continuada pode-se melhorar a qualidade da assistência e sugere-se também a criação de um Protocolo sobre a realização do contato pele a pele imediato, com o objetivo de padronizar esse contato na maternidade do estudo, pois ele é compreendido de diferentes formas pelos profissionais.

Ademais, pressupõe-se a necessidade de informar as mulheres sobre essa prática, seus benefícios, a maneira como deve ser realizada e o direito do acompanhante, assim como estimular a participação ativa no processo de parturição. O empoderamento das mulheres é essencial para a garantia de uma assistência qualificada e humanizada.

A limitação deste estudo é referente ao tempo do contato pele a pele, não foi possível avaliar essa variável, pelo fato de a maioria das puérperas não saber informar o tempo de contato e pela falta de registro no prontuário por parte dos profissionais. Outro aspecto importante que poderia interferir na realização do quarto passo da IHAC é o fato de a maternidade deste estudo ser referência em gestação de alto risco da microrregião, o que poderia evidenciar um maior número de complicações e intervenções durante o parto, contraindicando a realização dessa prática. Porém, nesta amostra a taxa de partos com problemas foi pequena e não foi associada ao contato pele a pele, não podendo, assim, ser considerado um fator limitante para a realização do contato pele a pele precoce.

Faz-se necessário realizar novas pesquisas sobre o assunto, pois até o momento de início do presente estudo, não existiam pesquisas relacionadas a esta temática no município de estudo, e foram poucas as pesquisas realizadas no território brasileiro. E ainda, é necessário compreender melhor os eventos e benefícios fisiológicos que o contato pele a pele pode acarretar no bebê e se ele realmente contribui para o processo de transição entre o meio intra e extrauterino.

\section{CONTRIBUIÇÕES DOS AUTORES}

Desenho do estudo: Raquel Elisabeth Cnossen. Lilian Fernandes Arial Ayres. Vanessa Doriguêtto Lima.

Coleta ou produção dos dados. Raquel Elisabeth Cnossen. Lilian Fernandes Arial Ayres. Brenda Alves Beirigo. Vanessa Doriguêtto Lima.

Análise de dados: Raquel Elisabeth Cnossen, Lilian Fernandes Arial Ayres, Camila Mendes dos Passos. Mara Rúbia Maciel Cardoso do Prado.

Interpretação dos resultados: Raquel Elisabeth Cnossen, Lilian Fernandes Arial Ayres e Camila Mendes dos Passos. Mara Rúbia Maciel Cardoso do Prado. 
Redação e revisão crítica do manuscrito. Raquel Elisabeth Cnossen. Lilian Fernandes Arial Ayres. Camila Mendes dos Passos. Vanessa Doriguêtto Lima. Mara Rúbia Maciel Cardoso do Prado. Brenda Alves Beirigo.

Aprovação da versão final do artigo. Raquel Elisabeth Cnossen. Lilian Fernandes Arial Ayres. Camila Mendes dos Passos. Vanessa Doriguêtto Lima. Mara Rúbia Maciel Cardoso do Prado. Brenda Alves Beirigo.

Responsabilidade por todos os aspectos do conteúdo e a integridade do artigo publicado. Raquel Elisabeth Cnossen. Lilian Fernandes Arial Ayres. Camila Mendes dos Passos. Vanessa Doriguêtto Lima. Mara Rúbia Maciel Cardoso do Prado. Brenda Alves Beirigo.

\section{EDITOR ASSOCIADO}

\author{
Gerson Luiz Marinho
}

\section{REFERÊNCIAS}

1. Moreira ME, Gama SG, Pereira AP, Silva AA, Lansky S, Souza Pinheiro $R$ et al. Clinical practices in the hospital care of healthy newborn infant in Brazil. Cad Saude Publica. 2014 ago;30(Suppl 1):S1-12. http://dx.doi. org/10.1590/0102-311X00145213. PMid:25167172.

2. Ministério da Saúde (BR), Secretaria de Atenção à Saúde, Departamento de Ações Programáticas Estratégicas. Atenção à saúde do recém-nascido: guia para os profissionais de saúde [Internet]. $2^{\mathrm{a}}$ ed. Brasília: Ministério da Saúde; 2014 [citado 2016 out 11]. 192 p. Disponível em: http://bvsms. saude.gov.br/bvs/publicacoes/atencao_saude_recem_nascido_v1.pdf

3. Barros GM, Dias MAB, Gomes SCS. O uso das boas práticas de atenção ao recém-nascido na primeira hora de vida nos diferentes modelos de atenção ao parto. Rev Soc Bras Enferm Ped. 2018;18(1):21-8. http:// dx.doi.org/10.31508/1676-3793201800004.

4. Lowson K, Offer C, Watson J, Mcguire B, Renfrew MJ. The economic benefits of increasing kangaroo skin-to-skin care and breastfeeding in neonatal units: analysis of a pragmatic intervention in clinical practice. Int Breastfeed J. 2015;10(11):11. http://dx.doi.org/10.1186/s13006015-0035-8. PMid:26000029.

5. Organização Mundial da Saúde, Fundo das Nações Unidas para a Infância. Iniciativa Hospital Amigo da Criança: revista, atualizada e ampliada para o cuidado integrado: modulo 3: promovendo e incentivando amamentação em um Hospital Amigo da Criança: curso de 20 horas para equipes de maternidade [Internet]. Brasília: Organização Mundial da Saúde; 2009 [citado 2016 out 12] (Série A. Normas e Manuais Técnicos). Disponível em: http://bvsms.saude.gov.br/bvs/publicacoes/ iniciativa_hospital_amigo_crianca_modulo3.pdf

6. World Health Organization. WHO recommendations: intrapartum care for a positive childbirth experience. Geneva: WHO; 2018.

7. Moore ER, Bergman N, Anderson GC, Medley N. Early skin-to-skin contact for mothers and their healthy newborn infants. Cochrane Database
Syst Rev.2016;11(11):CD003519. http://dx.doi.org/10.1002/14651858. CD003519.pub4. PMid:27885658.

8. Kologeski TK, Strapasson MR, Schneider V, Renosto JM. Contato pele a pele do recém-nascido com sua mãe na perspectiva da equipe multiprofissional. Rev enferm UFPE on line. 2017;11(1):94-101. http:// dx.doi.org/10.5205/reuol.9978-88449-6-1101201712.

9. Saxton A, Fahy K, Rolfe MI, Skinner V, Hastie C. Does skin-to-skin contact and breast feeding at birth affect the rate of primary postpartum haemorrhage: results of a cohort study. Midwifery. 2015;31(11):1110-7. http://dx.doi.org/10.1016/j.midw.2015.07.008. PMid:26277824.

10. dos Santos LM, da Silva JC, Carvalho ES, Carneiro AJ, de Santana RC, Fonseca MC.Vivenciando o contato pele a pele com o recém-nascido no pós-parto como um ato mecânico. Rev Bras Enferm. 2014;67(2):202-7. http://dx.doi.org/10.1590/0034-716719780002000007.PMid:24861061.

11. Pagano M, Gauvreau K. Princípios de bioestatística. São Paulo: Cengage Learning; 2010.

12. Organização Mundial da Saúde, Fundo das Nações Unidas para a Infância. Iniciativa Hospital Amigo da Criança: revista, atualizada e ampliada para o cuidado integrado: módulo 1: histórico e implementação [Internet]. Brasília: Organização Mundial da Saúde; 2008 [citado 2016 out 17]. (Série A. Normas e Manuais Técnicos). Disponível em: http:// www.redeblh.fiocruz.br/media/modulo1_ihac_alta.pdf

13. Bezerra FD, Menezes MAS, Mendes RB, Santos JMJ, Leite DCF, Kassar SB et al. Cuidado perinatal em um estado do nordeste brasileiro: estrutura, processos de trabalho e avaliação dos componentes do essential newborn care. Rev Paul Pediatr. 2019 abr/jun;37(2):140-8. PMid:30810691.

14. Castro RCM, Freitas CM, Damasceno AKC, Esteche CGE, Coelho TS, Brilhante AF. Resultados obstétricos e neonatais de partos assistidos. Rev enferm UFPE on line. 2018;12(4):832-9. https://doi.org/10.5205/19818963-v12i4a25202p832-839-2018.

15. Sampaio ÁRR, Bousquat A, Barros C. Skin-to-skin contact at birth: a challenge for promoting breastfeeding in a "Baby Friendly" public maternity hospital in Northeast Brazil. Epidemiol Serv Saude. 2016;25(2):281-90. PMid:27869946.

16. Lamounier JA, Bouzada MCF, Janneu AMS, Maranhão AGK, Araújo MFM, Vieira GO et al. Iniciativa Hospital Amigo da Criança, mais de uma década no Brasil: repensando o futuro. Rev Paul Pediatr. 2008;26(2):1619. http://dx.doi.org/10.1590/S0103-05822008000200012.

17. Carniel EF, Zanolli ML, Morcillo AM. Fatores de risco para indicação do parto cesáreo em Campinas (SP). Rev Bras Ginecol Obstet. 2007;29(1):34-40. http://dx.doi.org/10.1590/S0100-72032007000100006.

18. Haddad SM, Cecatti JG. Estratégias dirigidas aos profissionais para a redução das cesáreas desnecessárias no Brasil. Rev Bras Ginecol Obstet. 2011;33(5):252-62. PMid:21860933.

19. Diniz CSG, d'Orsi E, Domingues RMSM, Torres JA, Dias MAB, Schneck $\mathrm{CA}$ et al. Implementation of the presence of companions during hospital admission for childbirth: data from the Birth in Brazil national survey. Cad Saude Publica. 2014 ago;30(Suppl 1):S140-53. http://dx.doi. org/10.1590/0102-311X00127013. PMid:25167174.

20. D'Artibale EF, Bercini LO. The practice of the fourth step of the baby friendly hospital initiative. Esc Anna Nery Rev Enferm [Internet] 2014;18(2):356-64. http://dx.doi.org/10.5935/1414-8145.20140052. 